\title{
Noradrenaline depletion in patients with coronary artery disease before and after percutaneous transluminal coronary angioplasty with iodine-123 metaiodobenzylguanidine and single-photon emission tomography *
}

Christina Guertner ${ }^{1}$, Harald Klepzig Jr. ${ }^{2}$, Frank D. Maul ${ }^{1}$, Andreas Hartmann ${ }^{2}$, Sabolch Lelbach ${ }^{1}$, Alf Hellmann ${ }^{1}$, Gustav Hoer ${ }^{1}$

${ }^{1}$ Department of Nuclear Medicine, University Hospital, D-60590 Frankfurt/Main, Germany

${ }^{2}$ Department of Internal Medicine, Division of Cardiology, University Hospital, D-60590 Frankfurt/Main, Germany

Eur J Nucl Med (1993) 20:776-782

The headings in Table 1 were incorrectly placed.

The corrected table is published below

Table 1. Details of coronary artery stenoses before and after PTCA and corresponding MIBG uptake pattern

\begin{tabular}{llll}
\hline Patient & $\begin{array}{l}\text { Coronary artery } \\
\text { stenosis before PTCA }\end{array}$ & $\begin{array}{l}\text { Coronary artery } \\
\text { stenosis after PTCA }\end{array}$ & $\begin{array}{l}\text { MIBG uptake } \\
\text { pattern }\end{array}$ \\
\hline 1 & LCX $80 \%$ & LCX 20\% & $\begin{array}{l}\text { Re-innervation } \\
\text { Re-innervation }\end{array}$ \\
2 & LAD 90\% & LAD 20\% & Re-innervation \\
3 & RCA 99\% & RCA 30\% & Re-innervation \\
4 & LAD 70\% & LAD 30\% & Re-innervation \\
6 & RCA 90\% & RCA <40\% & No change \\
7 & LCX 70\% & LCX 40\% & No change \\
8 & RCA 90\% & RCA 40\% & No change \\
9 & RCA 99\% & RCA 50\% & No change \\
10 & LAD 99\% & LAD 99\% & Increased defect \\
11 & LAD 80\% & LAD 100\% & Increased defect \\
12 & LAD 70\% & LAD 90\% & Increased defect \\
13 & RCA 80\% $90 \%$ & No MIBG uptake due \\
& LAD 70\% diabetic polyneuropathy \\
14 & & LAD 20\% & (diabetes mellitus type II) \\
15 & RCA 70\% & & Globally reduced MIBG uptake \\
(diabetes mellitus type II)
\end{tabular}

